\title{
CHAPTER 6 \\ THE LATTICE SYSTEM AND THREE-DIMENSIONAL COMPUTER MODELING
}

\author{
Hiroaki Chiyolzura
}

15 he tremendous advances in computer technology in the last decade have brought the power of the mainframe computers of two decades ago to the desktop of today's computer user. We can easily find today extremely affordable space-saving mini- and midi-tower systems with central processing units (CPUs) with speeds of 1 $\mathrm{MHz}$ and upwards, with onboard random access memory (RAM) of $256 \mathrm{MB}$ with expansion easily managed, and data stored on hard disk drives of $40 \mathrm{~GB}$. We can see the results of our work on monitors which get physically smaller, while the diagonal screen size increases with sharper resolution. We can back up our data on rewritable CD-ROM's, $650 \mathrm{MB}$ capacity, and using readily-available digital ISDN and similar phone lines, we can distribute or share programs and data through an intranet to our local area networks, or internationally via the internet and the World-Wide Web (WWW).

The combination of all these factors allows us to develop an extremely high level of telecommunications for more and more comprehensive educational software. In particular, the intranet and WWW have opened up tremendous possibilities for the exchange and dissemination of medical, surgical and clinical information.

\section{1: Data Visualization}

The essential base of computer-assisted surgery and simulation is accurate visualization of clinical data, such as

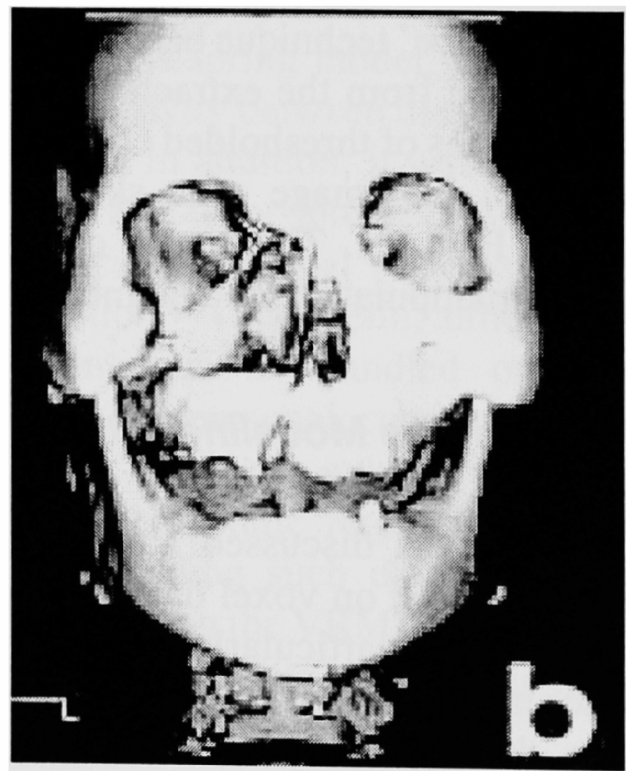

Fig 6.1: 2D scan images transformed to a 3D surface model. (a): typical CT scan of a head. By thresholding, i.e. assigning specific values to different densities in the scan image, specific components can be extracted from the slice, for example, bone. (b): Data from these extracted slices are converted into voxel images then 'stacked' in their original order, and by a process known as the 'marching cubes' method, they produce a surface rendered 3D image which can be rotated and manipulated in a variety of ways on a $2 \mathrm{D}$ computer monitor. The memory requirements are however very large. 
three-dimensional (3D) reconstructions of patient anatomy based on their actual two-dimensional (2D) radiographic images, which include amongst others computed tomography (CT), magnetic resonance imaging (MRI), magnetic resonance angiography (MRA) and positron emission tomography (PET) used individually or in combined imagery.

\subsection{1: $2 D$ images to $3 D$ modeling}

With CT scans, for example, serial scans are processed through a process called 'thresholding', in which values are assigned by the operator to different densities on each CT image. If the density of bone is assigned a certain value, and lower values or zero value assigned to any less dense portions of the image, then the computer can 'extract' only the bony tissue from each CT slice. Using the same process in MRI images, the computer can be programmed to select images with values lower than the value given to bone, and so a composite set of $2 \mathrm{D}$ soft tissue images can be built up.

The range of data from the serial slices is then expressed in voxels, and the three-dimensional image is built up using the 'marching cube' technique based on the voxels generated from the extracted serial data. Thus a series of thresholded CT slices can produce a $3 \mathrm{D}$ image of a skull, as illustrated in Figure 1. This image can be rotated and manipulated in a number of ways.

\subsection{2: $3 D$ Surface Modeling with Tissue Deformation}

The 3D images as discussed above are, however, still based on voxel data, but for realistic modeling, particularly of soft tissue, surface modeling is essential which incorporates the capability to deform the modeled tissue in a realistic manner. The current development of this stage of surface modeling has allowed us to use the computer images, in tandem with commercially-available software, to simulate complex surgical procedures in what we now

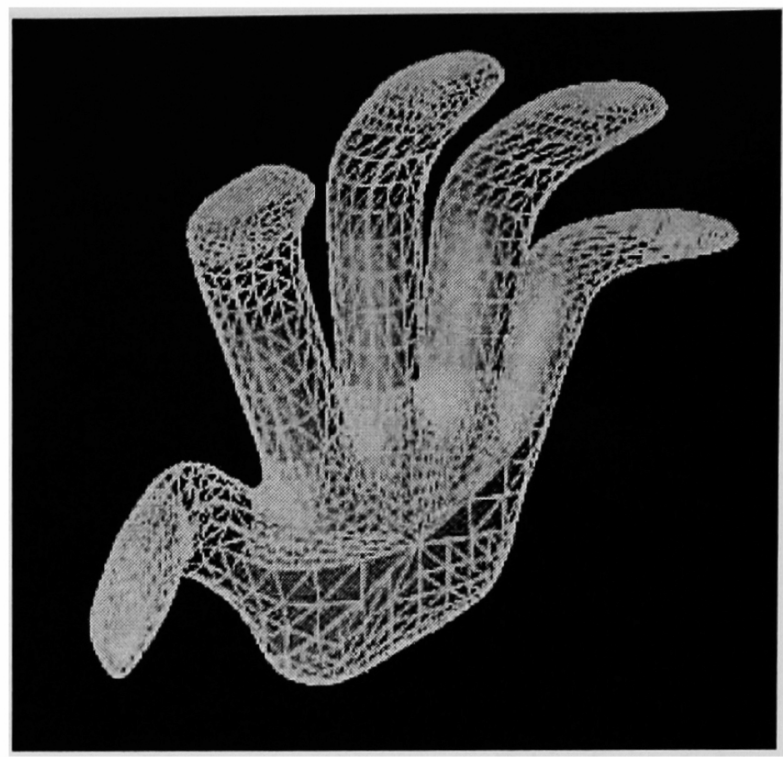

Fig 6.2: Polygon-based model of a hand (the surface rendering can be discerned beneath the polygon mesh). Each polygon is composed of a number of triangles, each of which requires a set amount of data for storage and manipulation. In order to produce a smooth surface, the number of polygons must be increased so the data requirements are enormous, and the surface of the hand model cannor be easily deformed. Because of the large memory requirements, even with high speed digital telecommunications it takes a long time to upload, send and download such images over the Net in the standard virtual reality modeling language (VRML) technique.

know as computer aided simulation surgery, or CASS. Good surface modeling must use another method, not voxel based, and until now in the field of computer graphics this has been based on the polygon method in which each polygon is constructed from a set of triangles. However, this method, although producing reasonable results, has two main disadvantages, The memory requirements for both data manipulation and image storage are huge, which is why earlier desk-top systems were unable to handle either the creation or the storage of polygon-based surface models, and the final results were not realistically smooth.

Figure 2 is an example of a hand modeled on the polygon system. Note the enormous number of data-crunching triangles. The smoother the surface has to be, the 
smaller the polygons must be, and thus the exponentially greater number of triangle sets required for each polygon impose massive memory requirements. When using comparatively expensive dedicated workstations with equally expensive sophisticated graphics capabilities, such as the SUN series of workstations used in conjunction with Silicon Graphics imaging systems, these memory-crunching polygon-based models could be used with great success, and some impressive results have been published and demonstrated at international medical imaging meetings.

One major problem still exists, however, in the physical memory needed for one single set of images, and their manipulation. In these days of telesurgery and telepresence, it is necessary to send these images over the web, using the virtual reality modeling language (VRML) technique. Even with high-speed digital telecommunications, a single image of several megabytes (MB) will take at least several minutes or even hours to send, and an equal time to download. Another major problem crops up when we try to deform specific tissue blocks in any of these polygon-based models to show the results of a craniomaxillofacial surgical procedure, for example, such as an osteotomy for the correction of a maxillofacial deformity. Not only does the 'solid' bone structure have to be simulated, but the overlying soft tissue must be deformed to allow the surgeon, and later the patient, to see the prospective result of the procedure. This capability of deforming tissue realistically becomes of essential importance when we are considering surgery on children, for whom the fourth dimension of time will also induce growth-related changes in both the skeletal and soft tissue structures, which must also be accurately modeled and simulated so that there are no unpleasant anatomical surprises deriving from the surgery, as the child, and his skeleton, grows.

Some reasonable deforming models have been developed, such as the Computer Aided Facial Expression Simulation (CAFES) system developed by Konno et $a l .{ }^{(1,2)}$ This system employs two polygon mesh models, one for the soft tissue and one for the underlying skeletal structures. Selected muscles were then modeled which connected the 'mobile' skin with the 'immobile' skeleton, and each muscle was assigned a value for tension, which moved the overlying soft tissue based on the mass and spring lattice model. By increasing or decreasing the value for the tension, the involved overlying soft tissue could be 'moved' in an appropriate manner. The disadvantage of the system, simplified though it is, lay in the huge memory requirements to handle the two polygon-based mesh systems for the skeletal and soft tissues, although it was comparatively user-friendly and interactive.

Another possibility was one of the mathematical deformable models, such as the finite element method, which allowed the exact mathematical description of nonlinear material properties, such as those associated with soft tissue. This method also was extremely CPU intensive, and unlike the mass-spring model, did not allow any interactivity between the model and the operator. In addition, it was extremely complex and labor-intensive to set up mathematically to model viscoelasticity plus relaxation. ${ }^{(3)}$ Once again, although these programs can be handled on present-day desk-top personal computer systems, the major problem exists of the enormous amount of time required in sending and downloading such data sets over the internet using the VRML technique, whereby the smallest data set is in the region of 10 MB.

\section{2: The Lattice Structure}

The mass-spring lattice system which was described above as used to allow the deformation of a polygon-based mesh model, gave an idea as to how a much simpler 


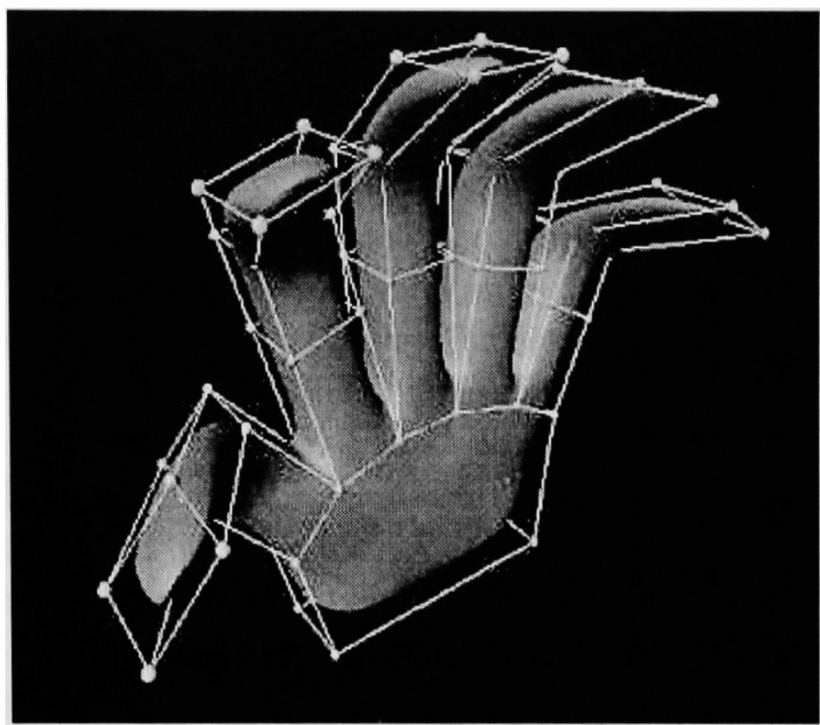

Fig 6.3: Lattice model of the same hand as in Figure 2 based on the Lattice structure (Lattice mesh and Lattice surface). The much simpler structure of the Lattice mesh, which is based on simplified polygons, can be seen: the Lattice mesh contains all the information necessary to create the Lattice surface. The center of each mesh plane is in contact with the surface of the model, and so manipulation of the plane's attributes and position will allow interactive and real-time manipulation of the model surface. Only the Lattice mesh is sent over the Net in what the author calls XVL (eXtended VRML with Lattice), and the memory requirements are much less.

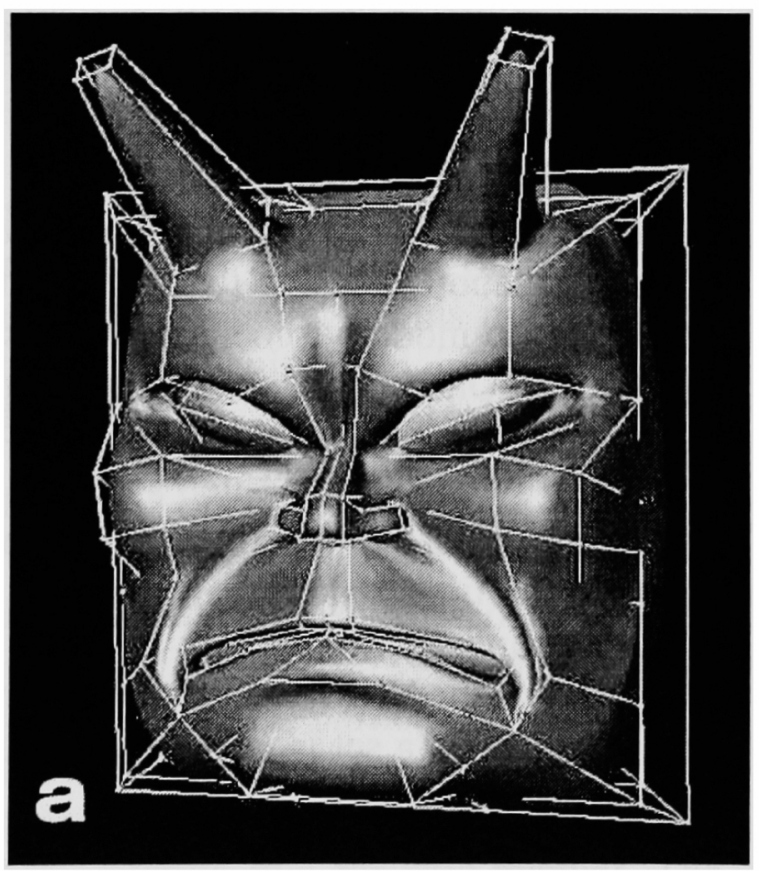

modeling system could be constructed, based on a simplified lattice network, which would simplify dramatically the process and thus reduce the memory requirements for deformable surface modeling. Thus the Lattice structure modeling method was developed and perfected by a Tokyo-based company of the same name, Lattice Technology. It offers smoother surfaces and better deformation, with very much simplified control of the surface model through the overlying Lattice mesh.

Basically, lattice modeling is based on a free-form surface technique the basics of which we originally described as far back as $1983,{ }^{(3)}$ in which the surfaces of interest are transferred and rendered with a limited data requirement. Figure 3 is a model of the same hand as in Figure 2, based on the lattice structure system. The center of each lattice plane contacts the surface of the model, and thus interactive deforming of the model is easily obtained by simply manipulating the respective lattice plane. We refer to this as XVL (eXtended VRML with

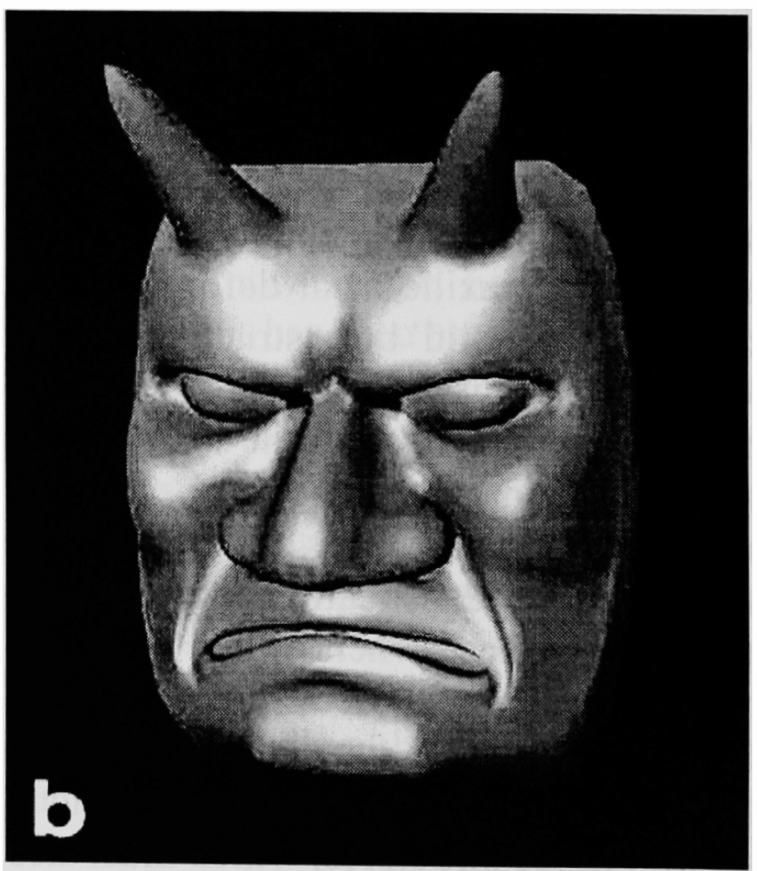

Fig 6.4: A surface model of a Japanese 'oni' (devil). (a): the Lattice mesh overlying the surface. (b): The final surface model. The surface is extremely smooth and very detailed, despite the obvious simplicity of the Lattice mesh. 
Lattice), allowing compact 3D data representation but with high quality, accurate and smooth surface rendering. ${ }^{(4)}$ The Lattice structure, which incorporates vertices, topologies and attributes, consists of the Lattice surface and Lattice mesh. The Lattice surface is a free-form surface area represented by a Gregory Patch, ${ }^{(4)}$ and the Lattice Mesh is a simplified polygon mesh which contains the properties of the surface data in vertices, topology and weighting properties. Only the Lattice mesh is transferred over the web in XVL, so the data requirements are minimized, and on downloading the Lattice mesh, the original Lattice surface can be quickly and accurately rendered, no matter how complex it is. Because XVL is a solid polygon model, it can also be used to send data to solid modeling machines to produce life-sized models for surgical simulation and education. Figure 4 is an example of the rendering from the Lattice structure (Lattice mesh and surface) to a smooth surface model.
To create a Lattice structure, the starting point is the original polygon-based mesh data, generated from a variety of sources such as CT, MRI and $3 \mathrm{D}$ surface scanning with laser or other light sources. The data are then compressed and converted into Lattice mesh, from which the Lattice surface is easily rendered. In Figure 5, featuring the same model as in Figure 4, Figure $5 \mathrm{a}$ is the polygon mesh model generated from a 3D surface scan by our own software. This model consists of 17,098 polygons requiring $85.9 \mathrm{MB}$ of memory. Our Lattice conversion software then compresses this (Figure 5b) into 1,899 polygons requiring only $93.1 \mathrm{kB}$ (i.e. 0.093 $\mathrm{MB}$ ), with a margin of error of less than $0.5 \%$, which we are currently trying to improve. It is however very similar to the error margin reported in the literature for polygon mesh generated models, when comparing measurements of the 3D screen-based models, solid life-sized models generated from the same data, and the topography of the original subject (Figure
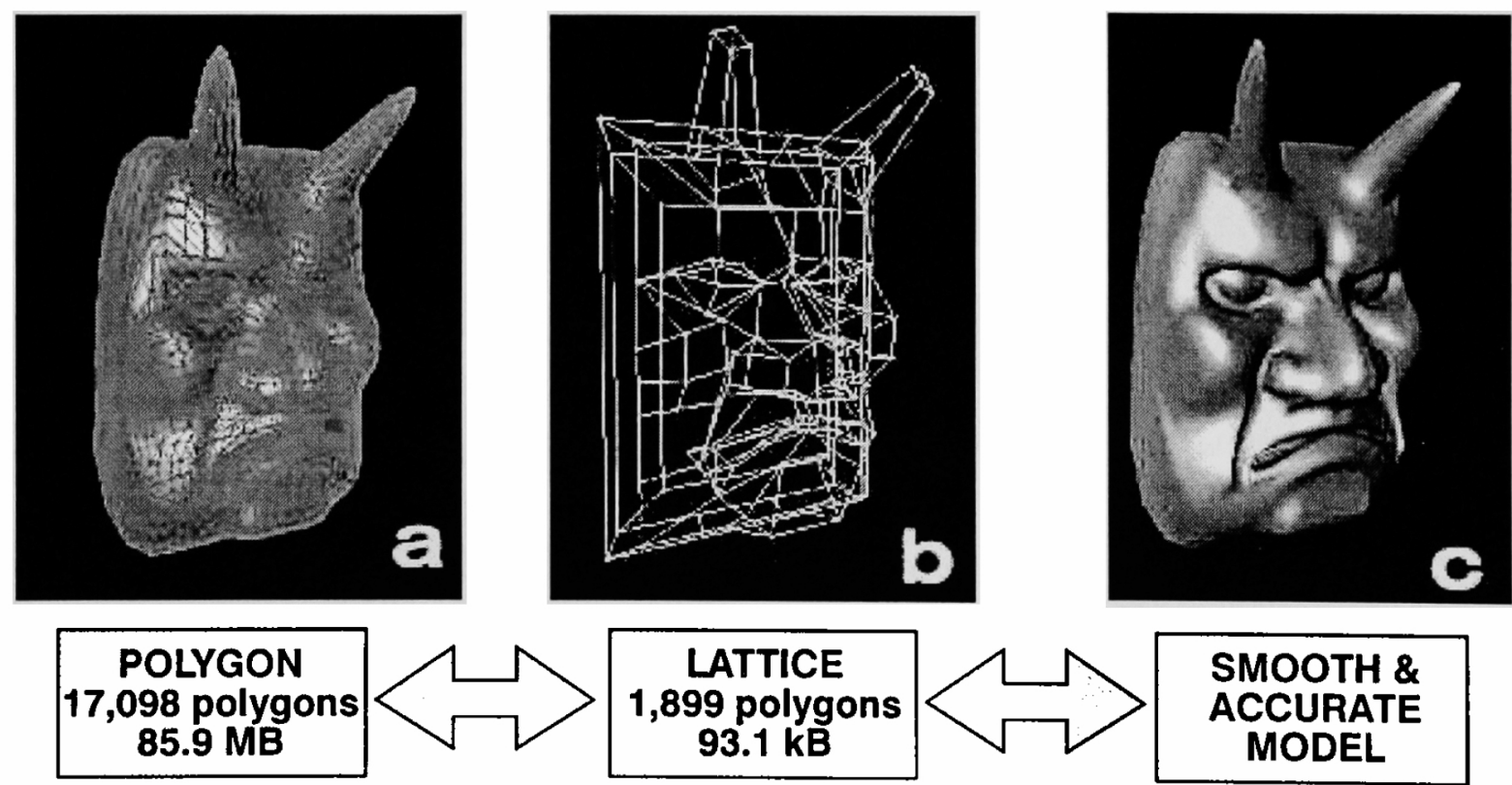

SMOOTH \& ACCURATE MODEL

Fig 6.5: The same model as in Figure 4, demonstrating the difference between conventional polygon and lattice mesh modeling memory requirements. (a): Polygon mesh model generated from a 3D surface scan, consisting of 17,098 polygons requiring $89.5 \mathrm{MB}$ of memory. (b): The polygon model is converted through our software program into Lattice mesh, requiring 1,899 polygons taking $93.1 \mathrm{kB}(0.093 \mathrm{MB})$ of memory. (c): The Lattice mesh contains all attributes of its associated surface, and easily and quickly generates a smooth, accurate 3D surface model. 
$5 c) \cdot{ }^{(6)}$

With Lattice structure, the applications in the medical field are very exciting. The surgeon can control the size of the data set, for example by selecting a specific region of interest from a full-face scan such as part of the lower mandible. Once selected, the data size drops, minimizing memory requirements while increasing the accuracy of the Lattice structure. This in turn offers precision control to increase accuracy of surgical simulation. Using XVL, these data sets can be quickly and easily sent over the WWW or intranet to colleagues for evaluation or instruction.

\section{3: Lattice Tools}

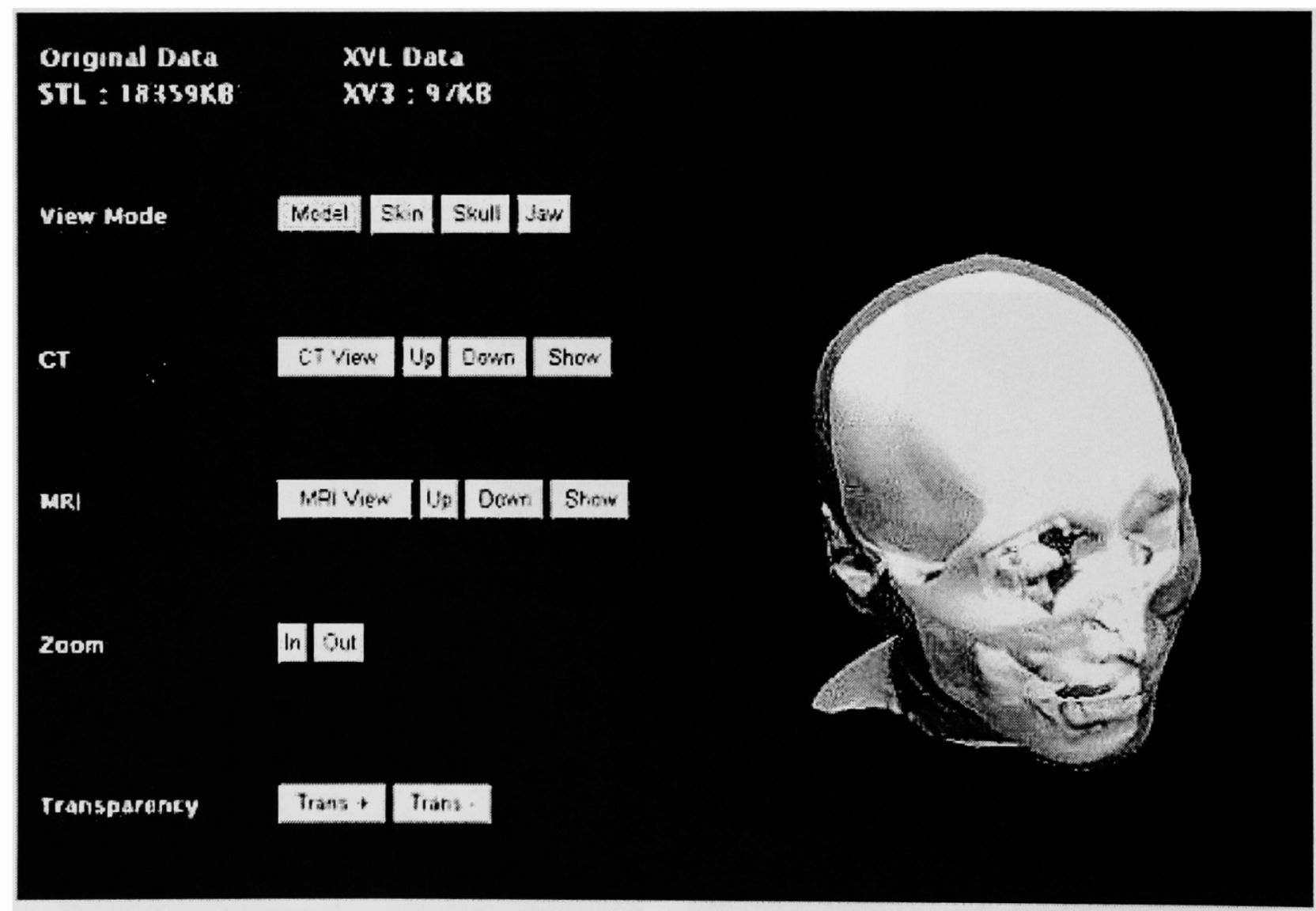

Fig 6.6: Screen shot captured from a Lattice structure model of a human head, generated from CT and MRI image data. The image can be rotated, skewed and manipulated very simply in real time using the mouse pointer. A selection of view modes can be selected of the model, the skin, the skull and the jaw, simply by clicking on the appropriate radio button. The original CT and MRI slice data are also maintained, so individual slices can be selected and shown. The image can be zoomed in or out, and the transparency of overlying structures can be interactively controlled in real time. When comparing the realistic volume rendering of the skull in this image with that in Figure 1, the verisimilitude of the Lattice structure model is very clearly superior. oped by Lattice Technology for 3D deformable modeling, and conversion between polygon mesh and Lattice mesh data.

\subsection{1: Lattice Designer}

Lattice designer is a comprehensive software program to develop structures, using interactive control of the Lattice planes. Existing models can be interactively deformed, or new models can be built from scratch. Computer requirements are very easily attained. The minimum processor is a Pentium II $200 \mathrm{MHz}$ or equivalent, although the higher the processor speed, the greater the accuracy and speed of the modeling and rendering. A minimum memory 
of $128 \mathrm{MB}$ and a $20 \mathrm{~GB}$ hard disk drive will give added speed and storage requirements, and although the small memory requirements of the Lattice structure make storage easy, if the user wishes to convert between polygon mesh and Lattice mesh, then the storage and handling requirements must be sufficient.

\subsection{2: Lattice Viewer}

With Lattice Viewer installed on his or her system, the medical professional can view 3D structures created in Lattice Designer, and sent over the internet or on CD-ROMs.

\subsection{3: Lattice Player}

Lattice structures can be easily deformatted interactively using Lattice Designer. Lattice Player offers an animation package to create, record and play back $3 \mathrm{D}$ animations based on Lattice structure data.

\subsection{4: Lattice Converter}

Lattice Converter is a software program which allows conversion between polygon mesh data and Lattice mesh data. Figure 6 is a screen shot captured from a Lattice structure representation of a human head. As can be seen, the original polygon mesh data required $18.4 \mathrm{MB}$, while the XVL data requirement is $97 \mathrm{kB}$, a very significant data reduction of over $99 \%$. A number of view modes are available, allowing real-time switching between surface model, involving the skin, and the underlying skull and jaw structures. Original CT and or MRI data may also be viewed, and the clinician can zoom in on specific structures or zoom out for the overall view, the transparency of which is also interactively controlled by the radio buttons at the bottom of the screen.

\subsection{5: Microsoft PowerPoint $₫$ Plug-In}

This software plug-in allows any image or animated series of images to be imported into Microsoft Corporations popular presentation preparation program, Power
Point $\AA^{\circledR}$. The potential for this plug-in for PC-based presentations is tremendous, as all the deformation and animation capabilities of Lattice Designer and Lattice Player are available to the presenter, including $360^{\circ}$ rotation and manipulation of the images.

\section{4: Conclusions}

Lattice structure brings interactive deformation of realistic 3D images into the desktop personal computer of the modern clinician, always provided the minimum requirements are met, which are at the time of writing extremely affordable. Within a year they will be even more affordable, given the current explosion of computer technology. We feel that Lattice structure, incorporating Lattice mesh and Lattice surface, is a revolutionary new program which, in addition to realistic and interactive 3D modeling, will allow faster and accurate transfer of clinical data over the web or intranet using our XVL technique, and equally speed up the downloading of images. The potential of Lattice structure in the increasing use of the WWW for telepresence, telesurgery and teleconsulting is enormous, as is its use in surgical simulation and education.

Further information on 3D web-based data can be found at the Keio University web site http://pegasus.sfc.keio.ac.jp, featuring educational software developed by our Keio team, or at Lattice Technologies home page at http://www.lattice.co.jp, which gives information on the company and the technology, as well as offering free evaluation downloads of Lattice software.

\section{References}

1: Konno $\mathrm{T}$, Mitani $\mathrm{H}$, Chiyokura $\mathrm{H}$ and Tanaka I (1996): Surgical simulation of facial paralysis. In Sieburg $\mathrm{H}$, Weghorst $\mathrm{S}$ and Morgan $\mathrm{K}$ (eds) Health Care in the Information Age. IOS Press and Ohmsha, 1996.

2: Keeve E, Girod S, Kikinis R and Girod B 
(1998): Deformable modeling of facial tissue for craniofacial surgery simulation. Computer Aided Surgery, 3: 228-238.

3: Chiyokura H and Kimura F (1983): Design of solids with free-form surfaces. Computer Graphics, 289-298.

4: Wakita A, Yajima M, Harada T, Toriya $\mathrm{H}$ and Chiyokura H (2000): XVL: A compact and qualified $3 \mathrm{D}$ represntation with lattice mesh and surface for the internet. Proceedings of VRML 2000. 45-51.

5: DeRose T, Kass $M$ and Truong T (1998): Subdivision surfaces in character animation. Computer Graphics.

6: Cavalcanti MGP, Haller JW and Vannier MW (1999): Three-dimensional computed tomographic landmark measurement in craniofacial surgical planning: experimental validation in vitro. Journal of Oral and Maxillofacial Surgery, 57: 690-694. 